\title{
A robust method for RNA extraction and purification from a single adult mouse tendon
}

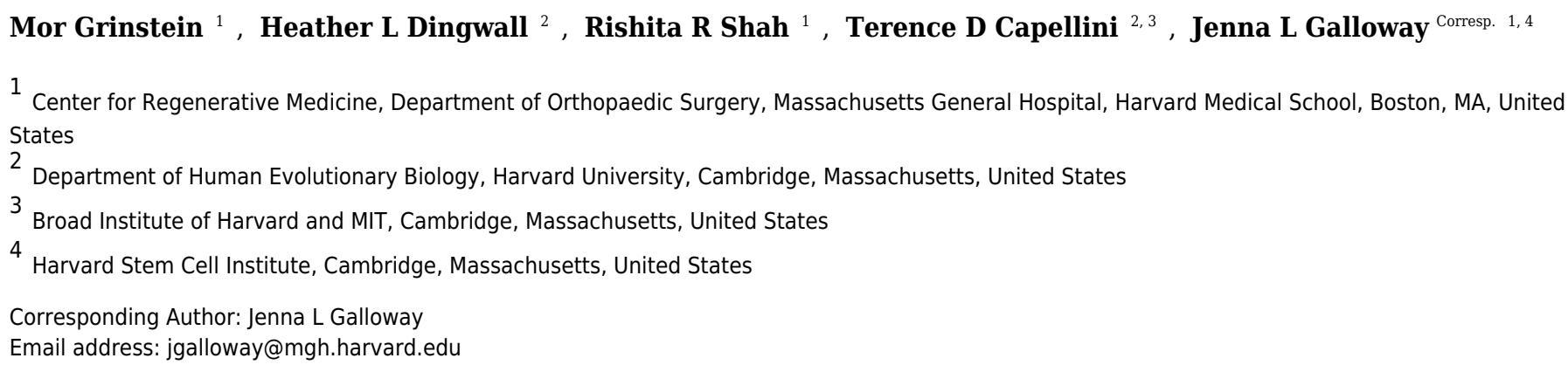

Background. Mechanistic understanding of tendon molecular and cellular biology is crucial towards furthering our abilities to design new therapies for tendon and ligament injuries and disease. Recent transcriptomic and epigenomic studies in the field have harnessed the power of mouse genetics to reveal new insights into tendon biology. However, many mouse studies pool tendon tissues or use amplification methods to perform RNA analysis, which can significantly increase the experimental costs and limit the ability to detect changes in expression of low copy transcripts. Methods. Single Achilles tendons were harvested from uninjured, contralateral injured, and wild type mice between 3-5 months of age, and RNA was extracted. RNA Integrity Number (RIN) and concentration were determined, and RT-qPCR gene expression analysis was performed. Results. After testing several RNA extraction approaches on single adult mouse Achilles tendons, we developed a protocol that was successful at obtaining high RIN and sufficient concentrations suitable for RNA analysis. We found that the RNA quality was sensitive to the time between tendon harvest and homogenization, and the RNA quality and concentration was dependent on the duration of homogenization. Using this method, we demonstrate that analysis of $S c x$ gene expression in single mouse tendons reduces the biological variation caused by pooling tendons from multiple mice. We also show successful use of this approach to analyze Sox9 and Col1a2 gene expression changes in injured compared with uninjured control tendons. Discussion. Our work presents a robust, cost-effective, and straightforward method to extract high quality RNA from a single adult mouse Achilles tendon at sufficient amounts for RT-qPCR as well as RNA-seq. We show this can reduce variation and decrease the overall costs associated with experiments. This approach can also be applied to other skeletal tissues, as well as precious human samples. 


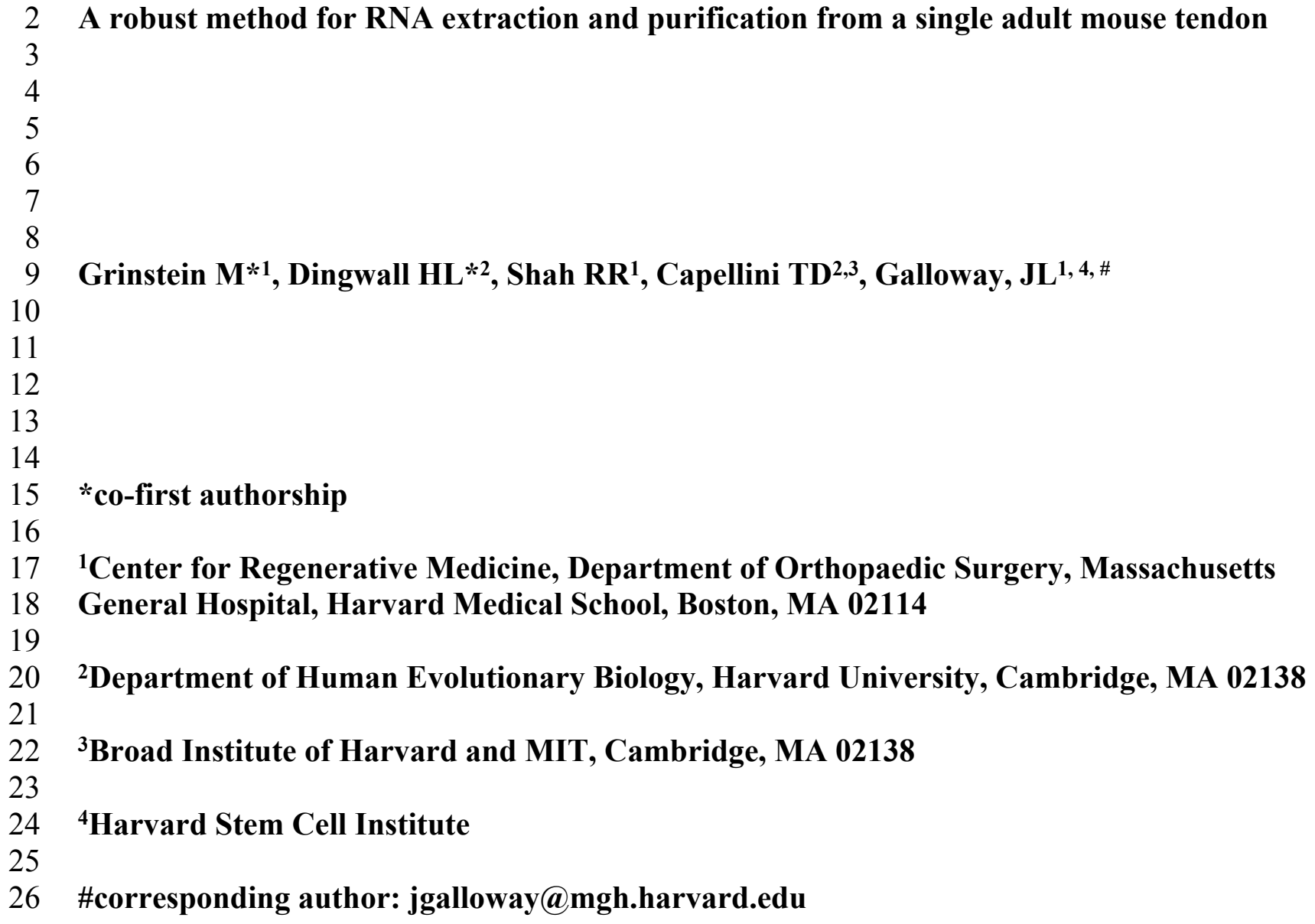

${ }^{3}$ Broad Institute of Harvard and MIT, Cambridge, MA 02138

${ }^{4}$ Harvard Stem Cell Institute

\#corresponding author: jgalloway@mgh.harvard.edu 
27 Abstract

28

Background. Mechanistic understanding of tendon molecular and cellular biology is crucial towards furthering our abilities to design new therapies for tendon and ligament injuries and disease. Recent transcriptomic and epigenomic studies in the field have harnessed the power of mouse genetics to reveal new insights into tendon biology. However, many mouse studies pool tendon tissues or use amplification methods to perform RNA analysis, which can significantly increase the experimental costs and limit the ability to detect changes in expression of low copy 35 transcripts.

Methods. Single Achilles tendons were harvested from uninjured, contralateral injured, and wild type mice between 3-5 months of age, and RNA was extracted. RNA Integrity Number (RIN) and concentration were determined, and RT-qPCR gene expression analysis was performed.

Results. After testing several RNA extraction approaches on single adult mouse Achilles tendons, we developed a protocol that was successful at obtaining high RIN and sufficient concentrations suitable for RNA analysis. We found that the RNA quality was sensitive to the time between tendon harvest and homogenization, and the RNA quality and concentration was dependent on the duration of homogenization. Using this method, we demonstrate that analysis of Scx gene expression in single mouse tendons reduces the biological variation caused by pooling tendons from multiple mice. We also show successful use of this approach to analyze Sox 9 and Colla2 gene expression changes in injured compared with uninjured control tendons.

Discussion. Our work presents a robust, cost-effective, and straightforward method to extract high quality RNA from a single adult mouse Achilles tendon at sufficient amounts for RT-qPCR as well as RNA-seq. We show this can reduce variation and decrease the overall costs associated with experiments. This approach can also be applied to other skeletal tissues, as well as precious human samples. 


\section{Introduction}

Tendon injuries are common problems for active individuals and the aging population (Kaux et al. 2011). Treatment options include physical therapy and surgical intervention, but pain and limited mobility often persist, making complete restoration of tendon function challenging (Nourissat et al. 2015). Our current understanding of the molecular and cellular pathways regulating tendons during homeostasis, healing, and aging are limited. Several studies using large animal models such as sheep, rabbits, and rats have provided important information about tendon injury, biomechanics, surgical techniques, and bioengineering strategies for tendon repair (Voleti 2012). Other studies have used mouse genetics to gain an understanding of the molecular and cellular response of tendons to acute injuries, changing load environments, and in gene loss-offunction models (Dunkman et al. 2014; Dyment et al. 2014; Howell et al. 2017; Mendias et al. 2008; Wang et al. 2017). The mouse system offers unique advantages for implementing mechanistic studies of tendon biology as they permit genetic lineage tracing and conditional knockout strategies, and they can be housed simply and in large numbers to improve sample sizes for functional studies. Even with inbred mouse strains, inter-animal variation can affect the conclusions drawn from gene expression analyses(Sultan et al. 2007; Watkins-Chow \& Pavan 2008). Therefore, the use of several biological replicates of tendon tissues obtained from individual mice for RNA analysis is essential for furthering our mechanistic understanding of tendon biology.

Mature tendons are comprised of type I collagen, which are arranged in a highly ordered hierarchical manner along the long axis of the tissue (Kannus 2000). Tendon cells lie between these organized fibrils and are surrounded by a hydrophilic, glycoprotein-rich ground substance (Bi et al. 2007; Kannus 2000; Yoon \& Halper 2005). This dense, fibrous, water-rich matrix that surrounds the tendon cells poses a significant challenge for the acquisition of high-quality RNA. In addition, tendons have low cell density compared with other tissues such as muscle or liver, resulting in minimal RNA yield per gram of tissue (Kannus 2000; Reno et al. 1997).

Previous studies have described protocols for RNA extraction from human or larger mammalian animal models such as rabbit (Ireland \& Ott 2000; Reno et al. 1997), but analyzing RNA from small animal models such as mouse can be more difficult. This issue has led to several different strategies for achieving RNA yield and quality sufficient for gene expression analysis by RT-qPCR or RNA-seq. RNA amplification methods have permitted gene expression analysis of single injured and uninjured tendons (Dunkman et al. 2014), but this can be prohibitively 
87 expensive for analyzing a large number of samples or target genes. In addition, studies in other 88 tissues have shown that such global pre-amplification can lead to biased results and increased false negative rates, especially for low- and medium-copy transcripts (Dunkman et al. 2014). Targeted 90 pre-amplification methods have been developed to minimize PCR bias by using multiplexed 91 primer pools at low concentrations combined with few PCR cycles (Jang et al. 2011). The resulting 92 amplified cDNA can be used either in SYBR Green-based or probe-based qPCR assays. While 93 such amplification methods have been shown to be highly sensitive and yield less-variable RT94 qPCR results compared to global cDNA amplification (Kroneis et al. 2017), the complex nature of the PCR amplification reactions imposes some limitations. First, targeted pre-amplification reactions require precision to yield usable cDNA. Because input sample concentrations, mRNA copy number, PCR cycle number, the specific combination of targets, and primer pool concentration can all affect the success of targeted pre-amplification, each assay must be individually optimized (Korenková et al. 2015; Kroneis et al. 2017). Improperly formulated reactions can lead to poor specificity and sensitivity of downstream qPCR, especially when using SYBR Green chemistry (Andersson et al. 2015). Such optimizations are time consuming and are not generalizable to different samples and different gene sets. Additionally, the nature of amplifying a specific set of targets inherently limits the possible downstream use of the preamplified samples.

Mendias and colleagues and Nielson and colleagues have performed gene expression analysis on a single mouse Achilles or plantaris tendon in different loss-of-function mouse models or in altered loading conditions (Mendias et al. 2008; Mendias et al. 2012; Nielsen et al. 2014)However, this approach is not widespread in the literature and these studies do not report on the RNA integrity, although they do report sample purity (260/280 ratio). However, there are examples of many studies that pool a large number of tendons (e.g., 12-20 individual tendons)

111 (Bell et al. 2013; Trella et al. 2017). Not only does this increase the mouse cohort size and

112 experimental costs, but it can also inflate the inter-individual variation, which may explain some 113 of the large variability in transcript abundance that was found in subsets of their gene expression 114 analysis (Trella et al. 2017). Lastly, other studies have focused on tendon-derived cell populations 115 such as tendon stem/progenitor cells (Bi et al. 2007). This approach results in robust RNA yields, 116 but it queries a cell population that has been expanded in culture and could have altered 117 transcriptomic and epigenomic states compared with that of native tendon tissue. 

using existing protocols enlarges the cohorts of mice needed for statistical analysis, and can hinder the use of RT-qPCR or functional genomic assays such as RNA-seq on single adult mouse tendons.

121 Here, we present a robust, low-cost, and straightforward RNA isolation protocol that enables the 122 isolation of high-integrity RNA from a single mouse Achilles tendon. We show that pooling tendon 123 samples inflates estimates of biological variance for gene expression data in RT-qPCR analysis.

124 We apply this method to analysis of injured and contralateral uninjured tendons to demonstrate the 125 detection of significant and reproducible gene expression changes. In addition, this method can be used to purify high quality RNA from other musculoskeletal tissues, making it easily adaptable to multiple connective and skeletal tissue types, or from difficult to obtain tissues from humans or other organisms.

\section{Methods}

\section{Mouse Studies}

134 Achilles tendons were collected from wildtype C57BL/6J mice between 3-5 months of age 135 (Jackson Laboratories 00664, $\mathrm{n}=30$ total). To compare gene expression levels between injured 136 and uninjured Achilles tendons in the same mouse, excisional Achilles tendon injuries were 137 performed using a $0.3 \mathrm{~mm}$ biopsy punch as described (Beason et al. 2012). The incision was closed 138 with 6-0 Ethilon nylon sutures and the tendons were harvested 30 days after injury for analysis.

139 Mice were housed, maintained, and euthanized according to American Veterinary Medical 140 Association guidelines. All experiments were performed according to our Massachusetts General 141 Hospital Institutional Animal Care and Use Committee (IACUC: 2013N000062) approved 142 protocol.

\section{RNA Extraction and Purification}

145 Dissected Achilles tendons were placed immediately into $1.5 \mathrm{ml}$ tubes containing $500 \mu 1$ of TRIzol 146 reagent (Invitrogen Cat\# 15596026) and high impact zirconium $1.5 \mathrm{~mm}$ beads (30-40 beads per 147 tube, D1032-15 Benchmark). Samples were homogenized in two 180-second rounds of bead 
148 beating at $50 \mathrm{~Hz}$ (BeadBug microtube homogenizer). Samples were then moved directly to dry ice 149 or $-80^{\circ} \mathrm{C}$ for longer storage up to 6 months.

150 Alternative tissue disruption procedures that were tested included homogenization of both

151 fresh and frozen tendons in $500 \mu \mathrm{l}$ TRIzol with a Polytron handheld homogenizer (PT 1200E, 152 Kinematica AG) until tissue was visibly disrupted (60 to 90 seconds). Cryogrinding of samples 153 was tested using a freezer mill (SPEX 6875). Achilles tendons were snap frozen in liquid nitrogen 154 and transferred to a super-cooled SPEX grinding cylinder (SPEX 6751C4) and pulverized in a bath 155 of liquid nitrogen for 3 minutes. Ground samples were collected by rinsing the cylinder with 500 $156 \mu \mathrm{l}$ TRIzol and transferred to a $1.5 \mathrm{ml}$ tube. For enzymatic digestion, tendons were placed in $2 \mathrm{ml}$ 157 Eppendorf tubes with $1 \mathrm{ml}$ digestion solution containing $0.2 \%$ collagenase II (Worthington, 158 LS004176) in DMEM (Gibco 11965) containing 0.1\% Penicillin/Streptomycin (Corning 30002cl) 159 and 1\% Hepes (Gibco 15630-080). Tubes were kept on ice during the dissection period and were 160 incubated together in a $37^{\circ} \mathrm{C}$ shaking water bath for 90 minutes. In order to digest remaining 161 matrix, we added $200 \mu \mathrm{L}$ of $0.2 \%$ collagenase I (Gibco 17100-017) and $300 \mu \mathrm{l}$ of $0.4 \%$ Dispase 162 (Gibco 17105-041) to the partially digested samples and incubated at $37^{\circ} \mathrm{C}$ for an additional 30 163 minutes. Following the digestion, the samples were centrifuged at $500 \mathrm{RCF}$ (g) for 5 minutes, the 164 supernatant was aspirated, and $500 \mu \mathrm{L}$ TRIzol was added. All homogenized samples were stored 165 at $-80^{\circ} \mathrm{C}$ until RNA isolation.

166 To extract RNA, the samples were thawed on ice followed by a 5-minute incubation at room temperature. Samples were quickly spun in the sample tubes and the homogenate was moved to a new Eppendorf tube, leaving behind the beads and residual tissue. Next, a chloroform extraction was performed, using double the recommended ratio of chloroform to TRIzol, which has been shown to increase RNA yield in small samples (Macedo \& Ferreira 2014). One hundred microliters of chloroform was added to the homogenate and vortexed well for approximately 1 minute. The TRIzol /chloroform mixture was then moved to a $1.5 \mathrm{ml} \mathrm{MaXtract} \mathrm{high} \mathrm{density} \mathrm{tube}$

173 (Qiagen Cat No. 129046), incubated at room temperature for 2-3 minutes, and spun $\geq 12,000 \times \mathrm{g}$ 174 at $4^{\circ} \mathrm{C}$ for 15 minutes. MaXtract tubes contain a sterile gel that forms a barrier between the RNA175 containing aqueous phase and the TRIzol/chloroform upon centrifugation at $4^{\circ} \mathrm{C}$, thus minimizing 176 carryover of organic solvents leading to an overall reduction in sample contamination. After 177 centrifugation, the aqueous phase was transferred to a clean $1.5 \mathrm{ml}$ Eppendorf tube and an equal 178 volume of $100 \%$ ethanol was added to the aqueous phase and mixed well. At this stage, the 
$179 \mathrm{RNA} /$ ethanol mix was typically stored at $-80^{\circ} \mathrm{C}$. We have found that brief incubation of this 180 mixture at $-80^{\circ} \mathrm{C}$ improved the total RNA yield, but it is not required.

181 RNA purification was next performed using the ZR Tissue \& Insect RNA MicroPrep kit

182 (Zymo Research R2030) or the Direct-Zol systems (Zymo Research R2050, R2060). Based on 183 typical tendon yields, the ZymoSpin IC spin columns are optimal for use with RNA extracted from 184 single tendons as these columns can purify up to $5 \mu \mathrm{g}$ of RNA in as little as $6 \mu$ l eluate. However, 185 this protocol also has been successfully used with ZymoSpin IIC columns, which require a larger 186 elution volume. After adding the RNA/ethanol mix to the spin column, the standard Zymo 187 purification protocol was used with the following modifications. First, a 15-minute on-column 188 DNase I treatment was added to minimize genomic DNA contamination. An extra wash step was 189 included to improve sample purity. Prior to elution, columns were spun for an additional 2 minutes

190 at maximum speed to remove residual ethanol. RNA was eluted in $15 \mu 1$ RNase/DNase free water 191 that was pre-warmed to $55-60^{\circ} \mathrm{C}$ to maximize the RNA recovery from the spin column. RNA 192 concentration was measured via fluorometric quantitation (Qubit HS RNA assay, Invitrogen, 193 CAT\# Q32852) and sample quality was determined by spectrophotometric analysis (NanoDrop 194 2000c, ThermoFisher Scientific) as well as capillary electrophoresis (2100 Bioanalyzer, Agilent).

195 The final RNA product was stored at $-80^{\circ} \mathrm{C}$ for RT-qPCR analysis.

196

\section{RT-qPCR, Data Analysis, and Statistics}

198 One hundred nanograms total RNA was reverse transcribed with oligo $(\mathrm{dT})_{20}$ primers using the 199 SuperScript IV First Strand Synthesis System (Thermo Fisher 18091050) and a no-reverse 200 transcriptase control was included for every sample. A total of $2 \mathrm{ng}$ cDNA template was amplified 201 for 40 cycles in each SYBR green qPCR assay (Applied Biosystems 4367659) using a final primer 202 concentration of $200 \mathrm{nM}$. All assays were performed in technical triplicate using either a LightCyclerII 480 (Roche; pooled samples) or a StepOnePlus Real Time PCR system (Applied Biosystems; injury samples). Three independent biological samples were run per condition for both sets of RT-qPCR. Gapdh was used as the reference gene for all samples (see Table 1 for 206 primer sequences).

All analyses were conducted in R 3.4.3 (R Core Team 2017). For the pooling experiment, summary statistics were calculated for Scleraxis $(S c x)$ and Gapdh technical and biological replicate cycle threshold $\left(\mathrm{C}_{\mathrm{T}}\right)$ values independently. Variance estimates for $\operatorname{Scx} \Delta \mathrm{C}_{\mathrm{T}}$ relative expression 
210 were calculated using standard error propagation techniques. Relative expression values for

211 Collagen Ia2 (Colla2) and SRY-Box9 (Sox9) were calculated for the injury analysis using the

$212 \Delta \Delta \mathrm{C}_{\mathrm{T}}$ method (Livak \& Schmittgen 2001) and injury samples were normalized to their

213 corresponding uninjured contralateral controls. Statistical differences between injured and

214 uninjured samples from three biological replicates $(n=3$ mice $)$ were analyzed via Welch's t-test

215 (Welch 1947) on the $\Delta \mathrm{C}_{\mathrm{T}}$ values.

216

217

218

219

\section{Results}

220

Several tissue disruption methods were tested in order to achieve optimal RNA quality and

221 quantity from a single mouse tendon. Among those tested were enzymatic digestion, cryogenic 222 grinding (manual and mill), shearing with a handheld homogenizer (i.e., rotor-stator), and bead beating. Capillary electrophoresis was performed on purified RNA using a Bioanalyzer RNA Nano chip (Agilent). RNA integrity number (RIN), a quantification of degradation, was calculated by the accompanying Agilent software based on the electropherogram for a given sample; a RIN of 10 indicates completely intact RNA whereas a RIN of 1 indicates severely degraded RNA. Enzymatic digestion produced intact RNA (RIN > 7), but low RNA yield $(\leq 1 \mathrm{ng} / \mu \mathrm{l})$. Cryogenic grinding and handheld homogenizer dissociation methods resulted in low yield $(\leq 5 \mathrm{ng} / \mu \mathrm{l})$ and poor RNA purity and integrity $(\mathrm{RIN} \leq 3)$ (Table $2 \mathrm{~A})$. Bead beater homogenization was found to produce the best results in terms of RNA quality (i.e., RIN $\geq 6.5$ ) and quantity ( $\geq 50 \mathrm{ng} / \mu \mathrm{l}$ ), and minimized carryover between samples. Additionally, bead beating was easily combined with standard TRIzol extraction and commercially available purification methods.

To further evaluate our bead beating homogenization method, we performed additional experiments examining the level of degradation that occurs prior to homogenization as well as during homogenization. To address the former, single Achilles tendons from similarly aged mice were left in sterile 1x PBS on ice following dissection for up to 9 minutes before homogenization to simulate waiting times involved in batch dissection. The shortest time between dissection and homogenization (0-30 seconds) yielded more intact RNA $(\mathrm{RIN}=6.5)$ while longer wait times resulted in more degraded RNA (9 minutes processing time RIN $=5.4$; Figure 1 ). This demonstrates that measurable degradation can occur prior to sample homogenization, and occurs with increases in time after dissection on the order of only minutes (Figure 1). Therefore, 
242 processing the tendon(s) immediately following dissection is essential for preserving RNA 243 integrity. We next tested how the duration of bead beating affects RNA quality by varying 244 homogenization times of single and four pooled Achilles tendons. Samples were homogenized for 24530 seconds, 60 seconds, 180 seconds, or 360 seconds (in two consecutive rounds of 180 seconds; 246 Figure 2 A, B). RNA from samples homogenized for less than 60 seconds suffered more 247 degradation than those that underwent longer homogenization times (Figure 2B), indicating 248 incomplete homogenization of the tissue during the shorter bead-beating periods. Homogenization 249 times of 720 seconds did not improve RIN numbers $(\mathrm{RIN}=6)$. This could be due to prolonging 250 the amount of time until RNA extraction or elevation of the temperature with longer 251 homogenization periods, leading to degradation. The temperature of TRIzol is a likely factor as 252 other studies with similar homogenization techniques used methods to lower its temperature (Leite 253 et al. 2012).

254 To test whether pooling tendons from multiple individuals into one sample prior to 255 homogenization influences RNA integrity, we measured RNA quality from single Achilles 256 tendons as well as pools of differing sizes ( $2,4,6$, and 8 tendons, $\mathrm{n}=3$ biological replicates per 257 pooling level; Figure 3 A, B). Electropherograms and RIN measurements show that RNA from all 258 pooling levels suffer levels of degradation similar to single Achilles samples (Figure 3A, B). Purity measurements were also similar among single and pooled samples (Table 2B). Therefore, pooling tendons from multiple individuals is not protective against RNA degradation; the only measure that improved with increased pool size was RNA yield (Figure 3C). To determine if pooling multiple samples affects gene expression measurements, we evaluated gene expression in single and differentially pooled tendon samples described above $(n=3$ per pooling level) via RT-qPCR. Although we find no gain in RNA quality from pooling, treating pools of tendons from multiple individuals as single biological replicates results in larger standard deviations in $\mathrm{C}_{\mathrm{T}}$ measurements in assays for Scx and Gapdh (Figure 4). This leads to larger sample variance for larger pools, driven

267 by differences in $\Delta \mathrm{C}_{\mathrm{T}}$ between biological replicates within a group, which impedes the detection 268 of small gene expression changes. Such increases in variance for pooled versus single samples 269 have also been reported for RNA-seq datasets (Rajkumar et al. 2015).

To validate the performance of the RNA obtained using this protocol, we performed RT271 qPCR for Sox9 and Colla2 expression on single Achilles tendons at 30 days following an acute 272 excision Achilles tendon injury. All samples were obtained from single injured and contralateral 
273 uninjured Achilles tendons from the same mouse. Using this protocol, we found significantly 274 increased expression of Sox9 and Colla2 in injured Achilles tendons compared with their 275 uninjured contralateral counterparts ( $\mathrm{p}<0.05$ for Sox 9 and $\mathrm{p}<0.01$ for Colla2; Figure 5). These 276 results are consistent with previous studies showing increased expression of Sox9 and Colla2 277 following tendon injury (Guerquin et al. 2013; Zhang \& Wang 2013), and also show that our 278 method is robust to detect gene expression changes in single tendon samples.

279

280

281

\section{Discussion and Conclusions}

282

283

284

285

286

287

288

289

290

291

292

293

294

295

296

297

298

299

300

301

302

Obtaining high quality RNA from tendons can be challenging, and this can limit the direction and scope of studies focused on analyzing adult mouse tendon tissues. Whereas a few studies have used single tendons without amplification, many other studies have used amplification or pooling of greater than 12 samples to detect gene expression changes. Both approaches can be expensive due to the high costs associated with amplification kits for multi-gene analysis or the number of mice used for one biological replicate. Dissociation, followed by culture and expansion of tendon-derived cells can yield greater RNA concentrations of high quality, but such approaches cannot be used to study gene expression changes after injury. The approach we described above provides a straightforward method to consistently obtain high yields of RNA from one Achilles tendon of sufficient quality to perform RT-qPCR analysis without amplification. Our reported RIN scores are also acceptable for standard RNA-seq differential expression analysis. In addition, studies have shown minimal variance among polyA-selected samples of high (RIN > 7) to moderate (RIN =6-7) RNA integrity as well as efficacy in correcting for variation in RNA integrity in the differential expression analysis (Jaffe et al. 2017; Romero et al. 2014). Although high RIN values should be the goal, there are some options for studies in which there are limitations in sample quality with moderate RIN scores. However, it must be noted that each RNA-seq library preparation system has specific input RNA requirements, and researchers should ensure that their RNA samples meet all manufacturer qualifications prior to use in a sequencing study.

Our analysis also uncovered key steps that are integral towards generating intact, high yield RNA from the single tendon samples. In particular, we find that the time from dissection to homogenization and storage can significantly impact the quality of the RNA, causing measurable 
304 degradation. In this regard, even small delays on the order of minutes could affect overall RNA 305 quality, which could greatly affect differential gene expression analysis. In addition, the duration 306 of homogenization is important for maximizing RNA yield and quality. Homogenization times 307 that are too short or long can result in dramatically different RIN and concentrations regardless of 308 the level of sample pooling.

309 Similar to previous RNA-seq studies, our RT-qPCR analysis of single and pooled tendon 310 samples revealed that pooling increases the variance of gene expression measurements (Rajkumar 311 et al. 2015). It has been argued that pooling samples from multiple individuals into single 312 biological replicates results in biological averaging and is therefore an appropriate, and even 313 useful, practice in gene expression studies via microarray (Kendziorski et al. 2005). However, 314 genes that are lowly expressed or exhibit subtle differences between conditions would require a 315 larger sample size of pools to achieve adequate statistical power, which would further inflate 316 mouse and reagent cost for RT-qPCR, microarray, or RNA-seq analyses (Shih et al. 2004). This 317 study also highlights the problem of performing RT-qPCR comparisons on a single pool per group 318 (run in technical triplicate), under the assumption that the within-sample variation is representative 319 of the biological variation among all animals of that group. Variance calculated from technical 320 replicates does not estimate biological variance within each group, and is not an appropriate 321 practice. Technical variation arises from noise due to measurement error and therefore is unrelated 322 to biological variation (Kitchen et al. 2010; Vaux et al. 2012), necessitating the use of multiple 323 pools for any statistical analysis.

324 Our tendon RNA extraction method is a robust protocol for obtaining high quality RNA

325 for gene expression assays. It decreases the number of mice required for analysis and avoids extra 326 amplification steps, making it straightforward, cost-effective, and easily accessible to researchers 327 new to the tendon field. By providing a means for reproducibly analyzing one Achilles tendon, 328 this method also reduces measurement error associated with pooling tendons from multiple 329 individuals. Moreover, our protocol permits the use of internal comparisons between a limb that 330 has undergone experimental manipulation (e.g., injury or unloading) and the contralateral control 331 limb within the same animal. In addition to facilitating larger-scale RT-qPCR studies, we believe 332 this method will make high dimensional gene expression analysis such as RNA-seq accessible to 333 more researchers studying musculoskeletal tissues, thus opening new frontiers in tendon biology. 334 
336 Acknowledgements: We would like to thank the Harvard University Bauer Core Facility and 337 MGH Center for Comparative Medicine for their services. 


\section{Figure Legends}

339

Figure 1. Length of time between dissection and processing affect RNA integrity

341 Electropherogram digital gel via Bioanalyzer shows integrity of RNA isolated from single Achilles

342 tendons that were kept on ice for various lengths of time $(0,3,6,9$ minutes) before

343 homogenization. All were homogenized for 360 seconds. Longer wait times prior to

344 homogenization reduce RNA quality. $18 \mathrm{~S}$ and $28 \mathrm{~S}$ are indicated and the green band is a marker.

Figure 2. Optimization of homogenization regime

347 Single Achilles tendons and pools of four tendons were subjected to different durations of bead 348 beating homogenization: 30, 60, 180, and 360 (in two rounds of 180) seconds. The 349 electropherogram digital gel shows that the longest beating time resulted in the most intact RNA, 350 as evidenced by the strong 28s and 18s bands with 360 seconds (A). RIN values called by Agilent software also show the improvement in quality with longer beating time (B).

352

\section{Figure 3. Tendon pooling affects RNA yield but not quality}

Representative electropherogram digital gel of RNA from different-sized pools of Achilles tendons

355

356

357

358

359

360

361

362

363

364

365

366

367

368 demonstrates high integrity RNA across all samples (A). Called RINs for pools ( $\mathrm{n}=3$ per pool) demonstrates that RNA quality from a single tendon is comparable to that from pools of tendons. Sample RINs are sufficiently high for use in RNA-seq gene counting and differential expression analysis for as low as one Achilles tendon (B). Concentration of RNA from single or pooled tendons increases with tendon number $(n=3$ per pool $)(C)$. The middle line represents the median, the box is quartiles 2 and 3 interquartile range (IQR), and whiskers are $1.5 \times \operatorname{IQR}(\mathrm{B}, \mathrm{C})$.

\section{Figure 4. Sample pooling affects estimates of sample variance in RT-qPCR}

$\mathrm{C}_{\mathrm{T}}$ standard deviations for $S c x(\mathrm{~A})$ and Gapdh (B) measurements were calculated for the technical replicates ( $n=3$ repeat measurements; dark grey) and for biological replicates $(n=3$ independent samples; light grey), separately. $\Delta \mathrm{C}_{\mathrm{T}}$ was calculated by normalizing $S c x \mathrm{C}_{\mathrm{T}}$ values to Gapdh. Technical (dark grey) and biological (light grey) variance estimates were calculated separately (C). All measures (A-C) show that biological variance increases as number of individuals contributing to a pool increases. 
369

370 Figure 5. Sensitivity and reproducibility of RT-qPCR on single tendon RNA

371 RT-qPCR of Sox9 (A) and Colla2 (B) expression of injured Achilles tendons relative to the 372 contralateral tendon of the same mouse at 30 days post-injury. A Welch's t-test shows that both 373 Sox9 and Colla2 expression was significantly different in the injured condition compared to the 374 control tendons ( $\mathrm{n}=3$ biological replicates, $\mathrm{p}<0.05$ for $\operatorname{Sox} 9$ and $\mathrm{p}<0.01$ for Colla2). 
376

377

378

379

380

381

382

383

384

385

386

387

388

389

390

391

392

393

394

395

396

397

398

399

400

401

402

403

404

405

406

407

408

409

410

411

412

413

414

415

416

417

418

419

420

\section{References}

Andersson D, Akrap N, Svec D, Godfrey TE, Kubista M, Landberg G, and Ståhlberg A. 2015. Properties of targeted preamplification in DNA and cDNA quantification. Expert review of molecular diagnostics 15:1085-1100.

Beason DP, Kuntz AF, Hsu JE, Miller KS, and Soslowsky LJ. 2012. Development and evaluation of multiple tendon injury models in the mouse. Journal of biomechanics 45:1550-1553.

Bell R, Li J, Gorski DJ, Bartels AK, Shewman EF, Wysocki RW, Cole BJ, Bach Jr BR, Mikecz K, and Sandy JD. 2013. Controlled treadmill exercise eliminates chondroid deposits and restores tensile properties in a new murine tendinopathy model. Journal of biomechanics 46:498-505.

Bi Y, Ehirchiou D, Kilts TM, Inkson CA, Embree MC, Sonoyama W, Li L, Leet AI, Seo BM, Zhang L, Shi S, and Young MF. 2007. Identification of tendon stem/progenitor cells and the role of the extracellular matrix in their niche. Nat Med 13:1219-1227.

Dunkman AA, Buckley MR, Mienaltowski MJ, Adams SM, Thomas SJ, Kumar A, Beason DP, Iozzo RV, Birk DE, and Soslowsky LJ. 2014. The injury response of aged tendons in the absence of biglycan and decorin. Matrix Biology 35:232-238.

Dyment NA, Hagiwara Y, Matthews BG, Li Y, Kalajzic I, and Rowe DW. 2014. Lineage tracing of resident tendon progenitor cells during growth and natural healing. PloS one 9:e96113.

Guerquin M-J, Charvet B, Nourissat G, Havis E, Ronsin O, Bonnin M-A, Ruggiu M, OliveraMartinez I, Robert N, and Lu Y. 2013. Transcription factor EGR1 directs tendon differentiation and promotes tendon repair. The Journal of clinical investigation 123:3564.

Howell K, Chien C, Bell R, Laudier D, Tufa SF, Keene DR, Andarawis-Puri N, and Huang AH. 2017. Novel Model of Tendon Regeneration Reveals Distinct Cell Mechanisms Underlying Regenerative and Fibrotic Tendon Healing. Scientific reports 7:45238.

Ireland ML, and Ott SM. 2000. The effects of pregnancy on the musculoskeletal system. Clinical orthopaedics and related research 372:169-179.

Jaffe AE, Tao R, Norris AL, Kealhofer M, Nellore A, Shin JH, Kim D, Jia Y, Hyde TM, and Kleinman JE. 2017. qSVA framework for RNA quality correction in differential expression analysis. Proceedings of the National Academy of Sciences:201617384.

Jang JS, Simon VA, Feddersen RM, Rakhshan F, Schultz DA, Zschunke MA, Lingle WL, Kolbert CP, and Jen J. 2011. Quantitative miRNA expression analysis using fluidigm microfluidics dynamic arrays. BMC genomics 12:144.

Kannus P. 2000. Structure of the tendon connective tissue. Scandinavian

Journal of Medicine and Science in Sports 10:312-320.

Kaux J-F FB, Goff CL, Crielaard J-M, Croisier J-L. 2011. Current Opinions on Tendinopathy. Journal of Sports Science \& Medicine 10(2):238-253.

Kendziorski C, Irizarry R, Chen K-S, Haag J, and Gould M. 2005. On the utility of pooling biological samples in microarray experiments. Proceedings of the National Academy of Sciences of the United States of America 102:4252-4257.

Kitchen RR, Kubista M, and Tichopad A. 2010. Statistical aspects of quantitative real-time PCR experiment design. methods 50:231-236.

Korenková V, Scott J, Novosadová V, Jindřichová M, Langerová L, Švec D, Šídová M, and Sjöback R. 2015. Pre-amplification in the context of high-throughput qPCR gene expression experiment. BMC molecular biology 16:5. 
421 Kroneis T, Jonasson E, Andersson D, Dolatabadi S, and Ståhlberg A. 2017. Global

422

423

424

425

426

427

428

429

430

431

432

433

434

435

436

437

438

439

440

441

442

443

444

445

446

447

448

449

450

451

452

453

454

455

456

457

458

459

460

461

462

463

464

465

466 preamplification simplifies targeted mRNA quantification. Scientific reports 7:45219.

Leite GM, Magan N, and Medina A. 2012. Comparison of different bead-beating RNA extraction strategies: an optimized method for filamentous fungi. J Microbiol Methods 88:413-418. 10.1016/j.mimet.2012.01.011

Livak KJ, and Schmittgen TD. 2001. Analysis of relative gene expression data using real-time quantitative PCR and the 2- $\Delta \Delta \mathrm{CT}$ method. methods 25:402-408.

Macedo NJ, and Ferreira TL. 2014. Maximizing Total RNA Yield from TRIzol Reagent Protocol: A Feasibility Study. ASEE Zone I Conference.

Mendias CL, Bakhurin KI, and Faulkner JA. 2008. Tendons of myostatin-deficient mice are small, brittle, and hypocellular. Proceedings of the National Academy of Sciences 105:388-393.

Mendias CL, Gumucio JP, Davis ME, Bromley CW, Davis CS, and Brooks SV. 2012. Transforming growth factor - beta induces skeletal muscle atrophy and fibrosis through the induction of atrogin - 1 and scleraxis. Muscle \& nerve 45:55-59.

Nielsen R, Clausen N, Schjerling P, Larsen JO, Martinussen T, List E, Kopchick J, Kjær M, and Heinemeier K. 2014. Chronic alterations in growth hormone/insulin-like growth factor-I signaling lead to changes in mouse tendon structure. Matrix Biology 34:96-104.

Nourissat G, Berenbaum F, and Duprez D. 2015. Tendon injury: from biology to tendon repair. Nature reviews Rheumatology 11:223-233.

Rajkumar AP, Qvist P, Lazarus R, Lescai F, Ju J, Nyegaard M, Mors O, Børglum AD, Li Q, and Christensen JH. 2015. Experimental validation of methods for differential gene expression analysis and sample pooling in RNA-seq. BMC genomics 16:548.

Reno C, Marchuk L, Sciore P, Frank CB, and Hart DA. 1997. Rapid isolation of total RNA from small samples of hypocellular, dense connective tissues. Biotechniques 22:1082-1086.

Romero IG, Pai AA, Tung J, and Gilad Y. 2014. RNA-seq: impact of RNA degradation on transcript quantification. BMC biology 12:42.

Shih JH, Michalowska AM, Dobbin K, Ye Y, Qiu TH, and Green JE. 2004. Effects of pooling mRNA in microarray class comparisons. Bioinformatics 20:3318-3325.

Sultan M, Piccini I, Balzereit D, Herwig R, Saran NG, Lehrach H, Reeves RH, and Yaspo M-L. 2007. Gene expression variation in Down's syndrome mice allows prioritization of candidate genes. Genome biology 8:R91.

Trella KJ, Li J, Stylianou E, Wang VM, Frank JM, Galante J, Sandy JD, Plaas A, and Wysocki R. 2017. Genome - wide analysis identifies differential promoter methylation of Leprel2, Foxf1, Mmp25, Igfbp6, and Peg12 in murine tendinopathy. Journal of Orthopaedic Research 35:947-955.

Vaux DL, Fidler F, and Cumming G. 2012. Replicates and repeats-what is the difference and is it significant? EMBO reports 13:291-296.

Voleti PB, Buckley, M. R. \& Soslowsky, L. J. n. . 2012. Tendon healing: repair and regeneratio. Annu Rev Biomed Eng 14:47-71.

Wang Y, Zhang X, Huang H, Xia Y, Yao Y, Mak AF-T, Yung PS-H, Chan K-M, Wang L, and Zhang C. 2017. Osteocalcin expressing cells from tendon sheaths in mice contribute to tendon repair by activating Hedgehog signaling. eLife 6.

Watkins-Chow DE, and Pavan WJ. 2008. Genomic copy number and expression variation within the C57BL/6J inbred mouse strain. Genome research 18:60-66.

Welch BL. 1947. The generalization ofstudent's' problem when several different population variances are involved. Biometrika 34:28-35. 
467 Yoon JH, and Halper J. 2005. Tendon proteoglycans: biochemistry and function. J Musculoskelet $468 \quad$ Neuronal Interact 5:22-34.

469 Zhang J, and Wang JH. 2013. The effects of mechanical loading on tendons-an in vivo and in vitro $470 \quad$ model study. PloS one 8:e71740.

471 
Table $\mathbf{1}$ (on next page)

RT-qPCR primer sequences 
1 Table 1. Primers used for RT-qPCR

\begin{tabular}{|l|l|l|}
\hline Gene & Forward Primer (5' to 3') & Reverse Primer (5' to 3') \\
\hline Sox 9 & AGTACCCGCATCTGCACAAC & TACTTGTAATCGGGGTGGTCT \\
\hline Colla 2 & CCAGCGAAGAACTCATACAGC & GGACACCCCTTCTACGTTGT \\
\hline Scx & AAGTTGAGCAAAGACCGTGAC & AGTGGCATCCACCTTCACTA \\
\hline Gapdh & TGTTCCTACCCCCAATGTGT & GGTCCTCAGTGTAGCCCAAG \\
\hline
\end{tabular}

2 


\section{Table 2 (on next page)}

Purity measurements for different homogenization methods and single vs pooled tendon samples

(A) RNA purity (260/280 ratio), concentration, and RIN measurements are poor following Polytron or Spex freezer mill homogenization methods. (B) Purity measurements are high regardless of pooling level with our bead beating protocol. 
1 Table 2. RNA purity measurements

2

3 A

\begin{tabular}{|c|c|c|c|c|c|c|}
\hline Sample & $\begin{array}{c}\text { Number } \\
\text { of } \\
\text { Tendons }\end{array}$ & $\begin{array}{c}\text { Conc. } \\
\text { (ng/ul) }\end{array}$ & $\begin{array}{c}\text { Homogenization } \\
\text { Method }\end{array}$ & RIN & $260 / 280$ & $260 / 230$ \\
\hline 8wk poly 1 & 1 & 32.5 & polytron & none & 1.57 & 0.49 \\
\hline 8wk poly 2 & 1 & 51 & polytron & 2.5 & 1.62 & 0.34 \\
\hline 8wk poly 3 & 3 & 71.2 & polytron & 2.6 & 1.58 & 0.33 \\
\hline 8wk poly 4 & 3 & 29.4 & polytron & none & 1.56 & 0.65 \\
\hline 8wk spex 1 & 4 & 170 & Spex freezer mill & 2.4 & 1.44 & 0.22 \\
\hline 8wk spex 2 & 4 & 80 & Spex freezer mill & 2.4 & 1.47 & 0.25 \\
\hline 8wk spex 3 & 1 & 4.4 & Spex freezer mill & 2.3 & 1.44 & 0.31 \\
\hline 8wk spex 4 & 1 & 4.7 & Spex freezer mill & none & 2.26 & 0.01 \\
\hline
\end{tabular}

4
5
6
7
8
9
10

B.

\begin{tabular}{|c|c|c|c|}
\hline Sample & $\begin{array}{c}\text { Number of } \\
\text { Tendons }\end{array}$ & $260 / 280$ & $260 / 230$ \\
\hline 1AT_1 & 1 & 2.06 & 1.97 \\
\hline 1AT_2 & 1 & 2.04 & 2.06 \\
\hline 1AT_3 & 1 & 2.01 & 2.03 \\
\hline 2AT_1 & 2 & 2.04 & 1.91 \\
\hline 2AT_2 & 2 & 1.99 & 1.88 \\
\hline 2AT_3 & 2 & 2.05 & 2.02 \\
\hline 4AT_1 & 4 & 2.06 & 2.05 \\
\hline 4AT_2 & 4 & 2.05 & 2.07 \\
\hline 4AT_3 & 4 & 2.05 & 2.06 \\
\hline 6AT_1 & 6 & 2.06 & 2.05 \\
\hline 6AT_2 & 6 & 2.06 & 2.05 \\
\hline 6AT_3 & 6 & 2.05 & 2.05 \\
\hline 8AT_1 & 8 & 2.07 & 2.08 \\
\hline 8AT_2 & 8 & 2.05 & 2.06 \\
\hline 8AT_3 & 8 & 2.06 & 2.05 \\
\hline
\end{tabular}




\section{Figure 1}

Length of time between dissection and processing affect RNA integrity

Electropherogram digital gel via Bioanalyzer shows integrity of RNA isolated from single Achilles tendons that were kept on ice for various lengths of time (0, 3, 6, 9 minutes) before homogenization. All were homogenized for 360 seconds. Longer wait times prior to homogenization reduce RNA quality. $18 \mathrm{~S}$ and $28 \mathrm{~S}$ are indicated and the green band is a marker. 


$$
\begin{aligned}
& \sum_{0}^{z} \sum_{m}^{z} \sum_{0}^{z} \sum_{\sigma}^{z}
\end{aligned}
$$

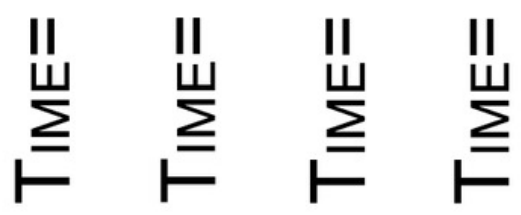

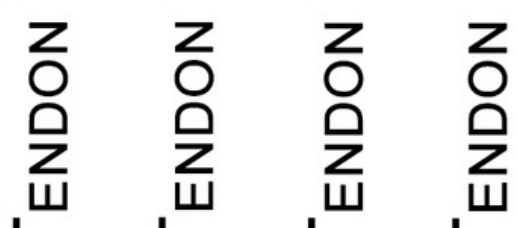

$$
\begin{aligned}
& \mapsto \stackrel{F}{\bullet} \\
& \leftarrow \leftarrow \leftarrow
\end{aligned}
$$

$6000 \mathrm{nt}$

$4000 \mathrm{nt}$
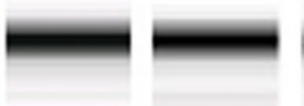

$28 S$

$2000 \mathrm{nt}$

$1000 \mathrm{nt}$

$500 \mathrm{nt}$

$200 \mathrm{nt}$

$25 \mathrm{nt}$

$$
\begin{aligned}
& \begin{array}{llll}
9 & 0 & + & + \\
0 & 0 & 0 & 0
\end{array}
\end{aligned}
$$

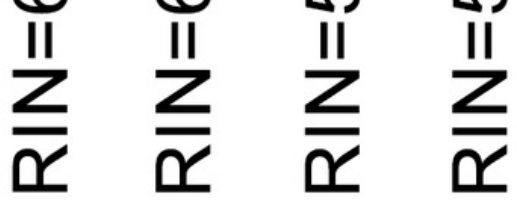




\section{Figure 2}

Optimization of homogenization regime

Single Achilles tendons and pools of four tendons were subjected to different durations of bead beating homogenization: 30,60, 180, and 360 (in two rounds of 180) seconds. The electropherogram digital gel shows that the longest beating time resulted in the most intact RNA, as evidenced by the strong $28 \mathrm{~s}$ and $18 \mathrm{~s}$ bands with 360 seconds (A). RIN values called by Agilent software also show the improvement in quality with longer beating time (B). 
A
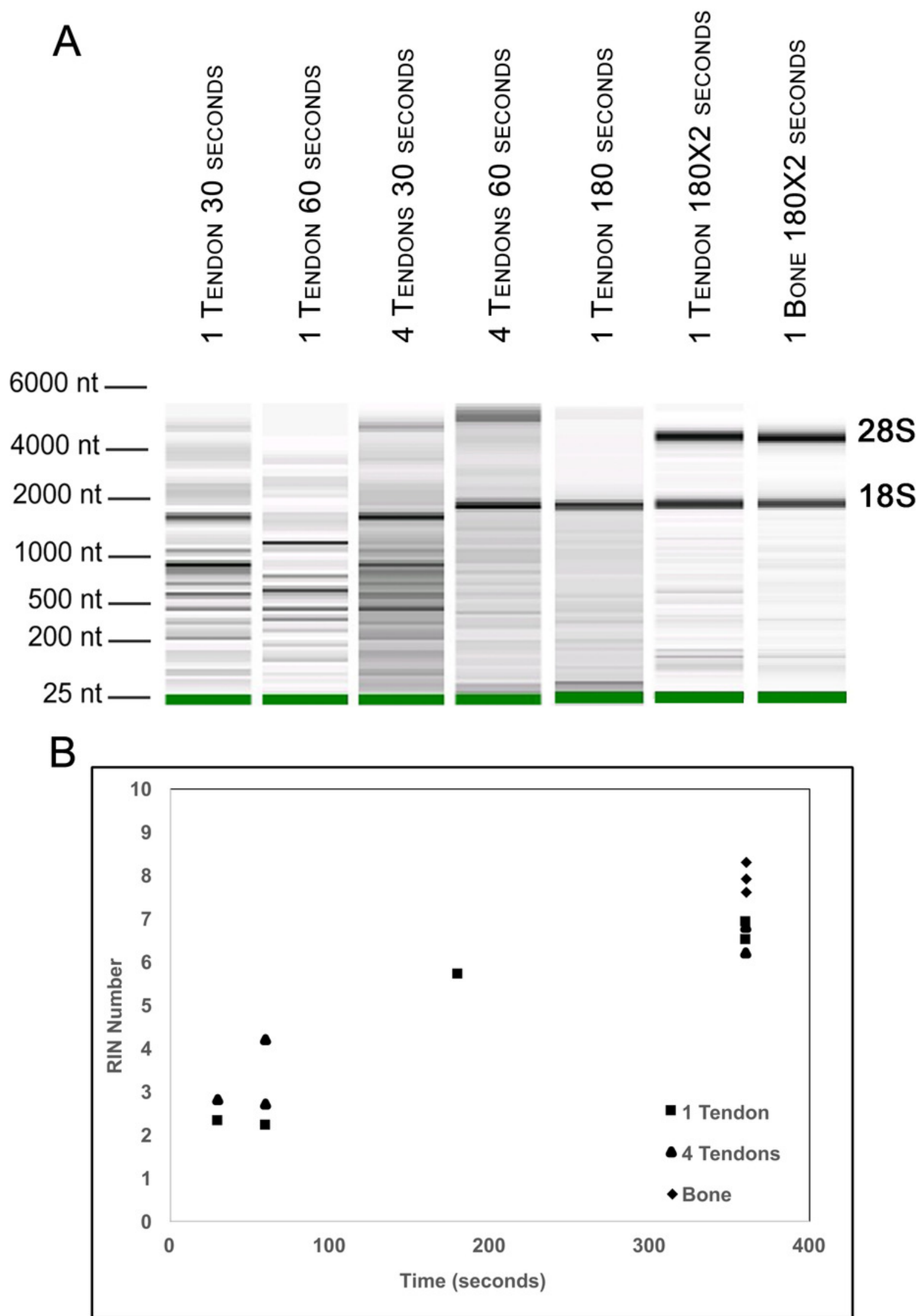


\section{Figure 3 (on next page)}

Tendon pooling affects RNA yield but not quality

Representative electropherogram digital gel of RNA from different-sized pools of Achilles tendons demonstrates high integrity RNA across all samples (A). Called RINs for pools ( $n=3$ per pool) demonstrates that RNA quality from a single tendon is comparable to that from pools of tendons. Sample RINs are sufficiently high for use in RNA-seq gene counting and differential expression analysis for as low as one Achilles tendon (B). Concentration of RNA from single or pooled tendons increases with tendon number ( $n=3$ per pool) (C). The middle line represents the median, the box is quartiles 2 and 3 interquartile range (IQR), and whiskers are $1.5 \times$ IQR $(B, C)$. 


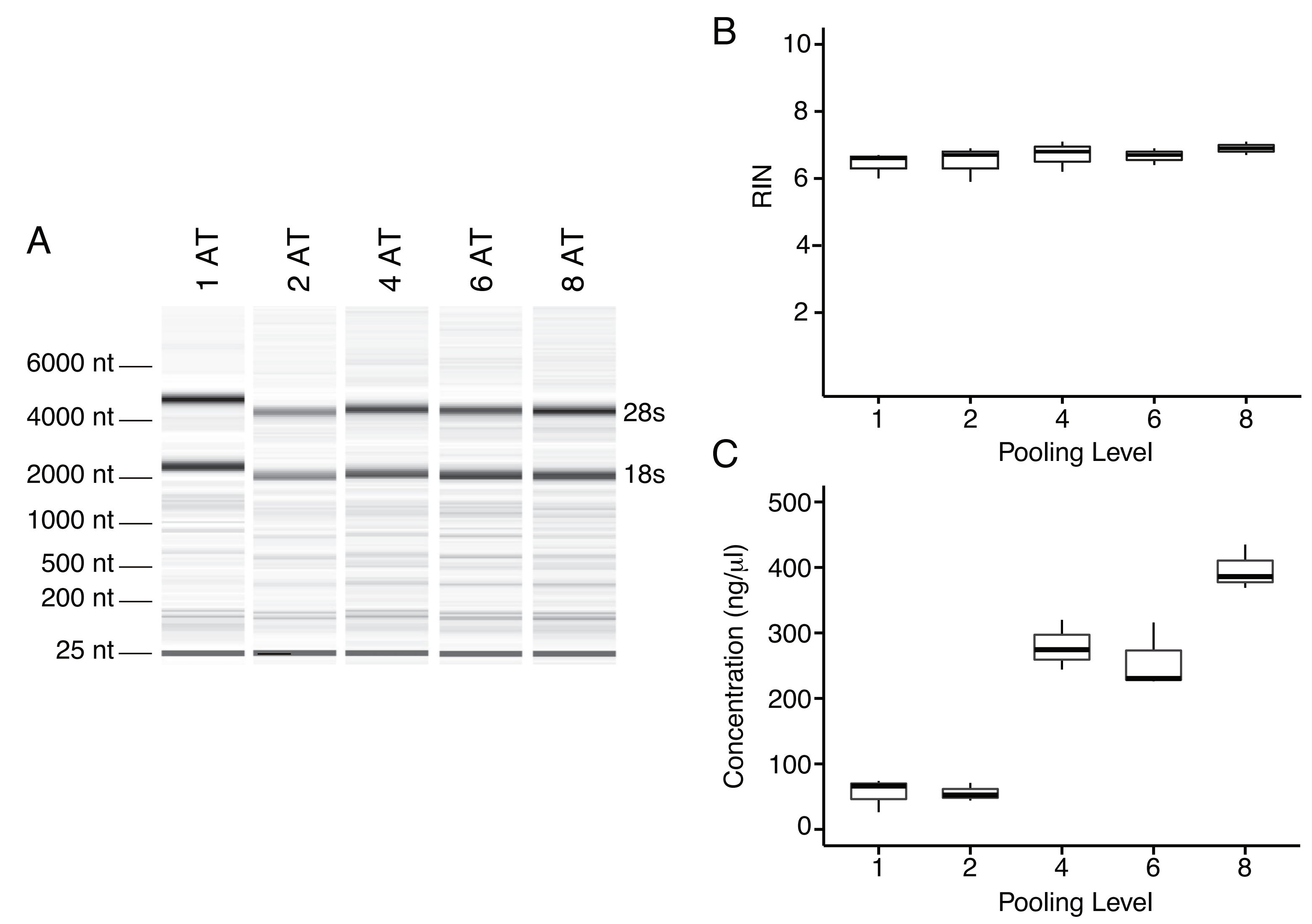


Figure 4 (on next page)

Sample pooling affects estimates of sample variance in RT-qPCR

$\mathrm{C}_{\mathrm{T}}$ standard deviations for Scx (A) and Gapdh (B) measurements were calculated for the technical replicates ( $n=3$ repeat measurements; dark grey) and for biological replicates ( $n$ $=3$ independent samples; light grey), separately. $\Delta C_{T}$ was calculated by normalizing $S c x C_{T}$ values to Gapdh. Technical (dark grey) and biological (light grey) variance estimates were calculated separately (C). All measures (A-C) show that biological variance increases as number of individuals contributing to a pool increases. 
Figure $\mathbf{5}$ (on next page)

Sensitivity and reproducibility of RT-qPCR on single tendon RNA

RT-qPCR of Sox9 (A) and Col1a2 (B) expression of injured Achilles tendons relative to the contralateral tendon of the same mouse at 30 days post-injury. A Welch's t-test shows that both Sox9 and Colla2 expression was significantly different in the injured condition compared to the control tendons ( $n=3$ biological replicates, $p<0.05$ for Sox 9 and $p<0.01$ for Colla2). 
A

Pexp

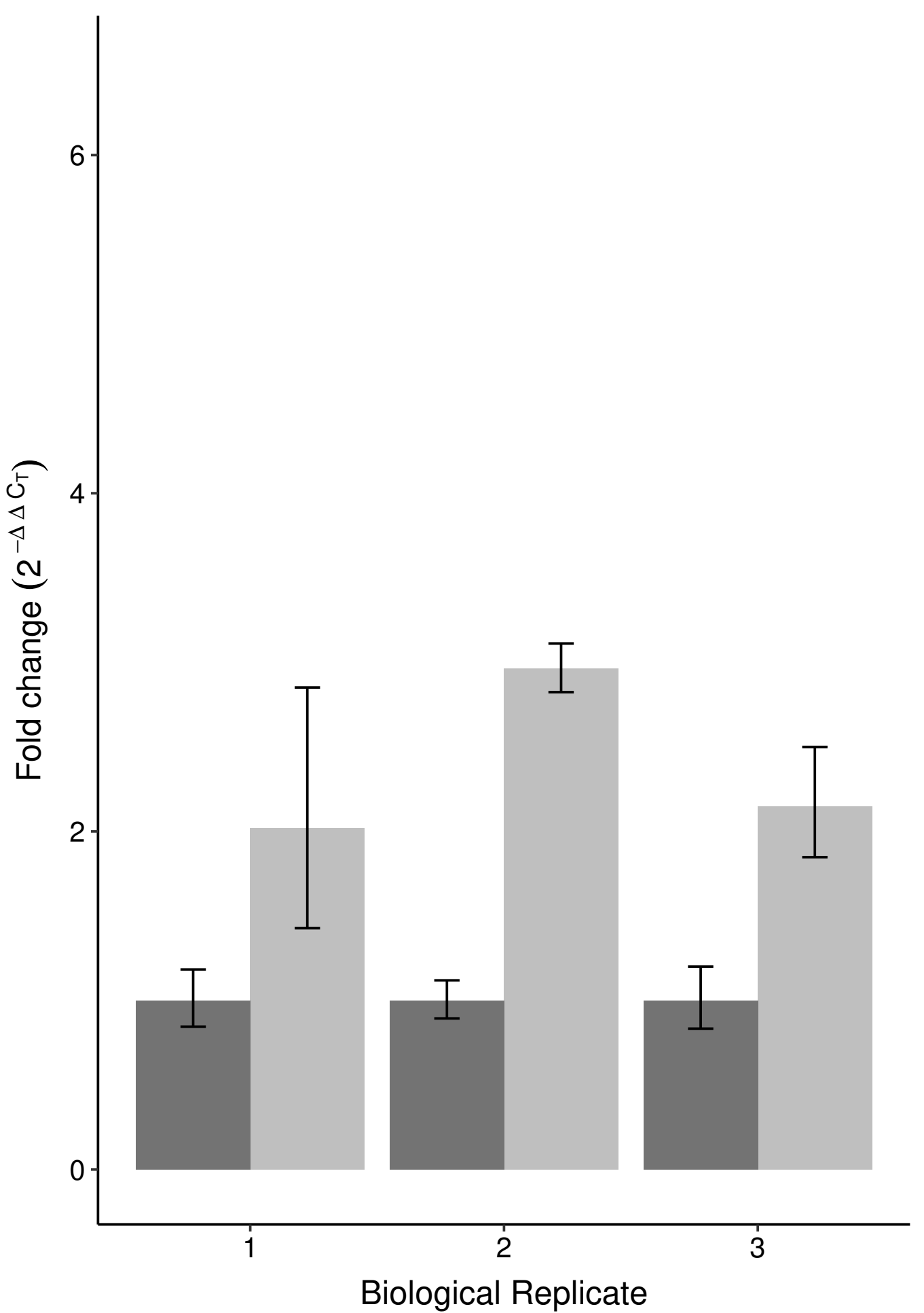

B Manuscript to be reviewe Col1 a2

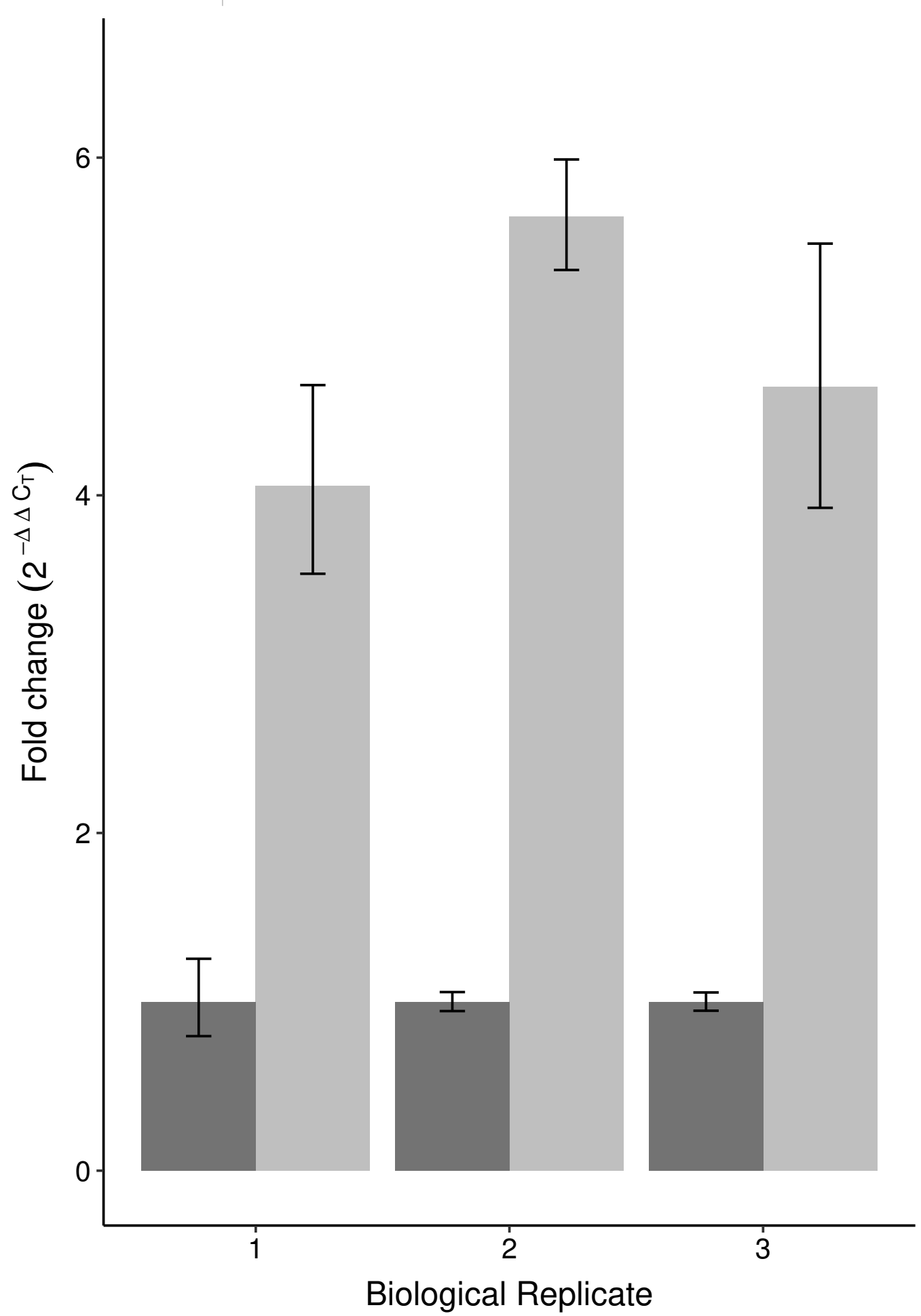

